\title{
ADAPTABILIDAD DE SEIS CULTIVARES DE CHILE DULCE BAJO INVERNADERO EN GUANACASTE
}

\section{RESUMEN}

Se evaluaron seis cultivares de chile dulce (Capsicum annum), en la Estación Experimental Enrique Jiménez Núñez (EEEJN), ubicada en Cañas, Guanacaste, durante los meses de octubre del 2014 a mayo del 2015. Todos los cultivares evaluados presentaron frutos de tipo cónico y con maduración a rojo. Los cultivares que obtuvieron los mayores rendimientos productivos fueron FBM 10 y Dulcitico con 68,10 y $56,87 \mathrm{t} / \mathrm{ha}$ respectivamente, sin que mostraran diferencias significativas entre ellos. Todos los materiales a excepción de Dulcitico obtuvieron una mayor proporción de frutos de primera (longitud fruta $\geq 10 \mathrm{~cm}$ ) que de segunda calidad (longitud fruto $<10 \mathrm{~cm}$ ). El cultivar FBM 10 presentó un 52,02 \% de frutos de primera, con un $47,35 \%$ de frutos de segunda y un $0,63 \%$ de tercera (no comercial). Las condiciones climáticas más drásticas dentro del invernadero se presentaron a la 1 p.m., en promedio la temperatura fue de 35,1 ${ }^{\circ} \mathrm{C}$, la humedad relativa llegó a $54,2 \%$ y la radiación fue de 98 watts $/ \mathrm{m}^{2}$. El rubro que obtuvo el mayor costo económico fue el invernadero con 1,5 millones de colones por $1000 \mathrm{~m}^{2}$, lo que significa un $50 \%$ de la inversión inicial. El cultivar de chile dulce que obtuvo la mayor utilidad neta, fue el FBM 10 con 2,11 millones de colones por cada $1000 \mathrm{~m}^{2}$. Esto se debió a que obtuvo los mayores ingresos totales de frutos de primera y segunda, con 3,08 y 2,10 millones de colones respectivamente, superando en ingresos totales al cultivar testigo (Nathalie) por 1,08 millones de colones $/ 1000 \mathrm{~m}^{2}$.

Palabras clave: ambiente protegido, híbridos, hidroponía, factores climáticos, relación beneficio costo.

\section{INTRODUCCIÓN}

El chile (Capsicum sp) es uno de los cultivos hortícolas más importantes del mundo, sembrándose en el año 2013 una superficie de 1,9 millones de hectáreas, con una producción de 31,2 millones de toneladas. Entre los países productores más importantes destacan China y México con 16,0 y 2,3 millones de toneladas respectivamente (FAO 2014). Actualmente el chile dulce es uno de

\begin{abstract}
los vegetales preferidos por la población costarricense, alcanzándose en el año 2012 un consumo per cápita de $3,3 \mathrm{~kg}$ (PIMA-CENADA 2013). Entre las cualidades que destacan los consumidores se encuentran su alto valor nutritivo y la multiplicidad de usos, tanto en consumo fresco, como en productos industriales, congelados, pastas, deshidratados y salsas (Samaniego 2006).
\end{abstract}

1 Instituto Nacional de Innovación y Transferencia en Tecnología Agropecuaria. INTA. Costa Rica. rramirez@inta.go.cr, jaguilar@inta.go.cr y Imeza@inta.go.cr. Sede de la Estación Experimental Enrique Jiménez Núñez del INTA. Cañas, Guanacaste. 
Esta planta es monoica, de ciclo anual y puede alcanzar entre $0,6 \mathrm{~m}$ a $1,5 \mathrm{~m}$ de altura, dependiendo tanto de la variedad, como de las condiciones climáticas y de manejo (Orellana et al. 2012). La actividad fotosintética tiene una clara respuesta a la temperatura, mostrando un mínimo sobre los $5^{\circ} \mathrm{C}$ y un óptimo entre 25 a $35^{\circ} \mathrm{C}$ en la mayoría de las especies hortícolas, disminuyendo a valores superiores (Urban 1997). En Costa Rica se cultiva en zonas con temperaturas entre los 18 y $30^{\circ} \mathrm{C}$, aunque gracias a las mejoras en el nivel tecnológico transferido a los productores, las áreas de siembra se han expandido prácticamente a todo el territorio nacional (Samaniego 2006).

Una de las debilidades que presenta esta actividad en nuestro país, es la escasa oferta de germoplasma tolerante a las condiciones de alta temperatura, siendo el híbrido Nathalie uno de los más utilizados por los productores. Aunado a esta problemática también se suma el bajo rendimiento promedio nacional que es de 15 t/ha, significando un promedio de 0,6 kg/planta (Morales y Rodríguez 2010). No obstante, esto contrasta con los resultados obtenidos en la Estación Experimental Agrícola Fabio Baudrit Moreno (EEAFBM) en el año 2012, donde se alcanzaron rendimientos de 95,5 t/ha con el híbrido Dulcitico (Echandi, 2012) y por los obtenidos en este mismo periodo por el Instituto Nacional de Innovación y Transferencia en Tecnología Agropecuaria (INTA) en la Región Chorotega (41 t/ha), con el híbrido Andes Cobayashi (Ramírez et al. 2013).

Un aspecto que se debe tomar en cuenta a la hora de cuantificar la producción es clasificarla según las categorías comerciales del mercado, ya que cada una de estas se paga en forma distinta. Según el reglamento técnico para el consumo fresco de chile dulce, publicado en el Diario Oficial La Gaceta, se consideran frutos grandes, aquellas que superan los $15 \mathrm{~cm}$ de longitud, medianos de 10 a $15 \mathrm{~cm}$ y pequeños los menores a $10 \mathrm{~cm}$ (Ministerio de Salud et al. 2007). A nivel comercial de supermercados, los frutos grandes y medianos se catalogan como primera calidad, los pequeños como segunda calidad y los frutos con defectos o quemaduras de sol, como tercera calidad, las cuales no tiene valor monetario.

El fenotipo puede cambiar por variaciones ambientales, por lo que la evaluación de cultivares en diferentes sitios, permite recomendar aquellos que posean un mejor comportamiento en una región determinada (Giménez et al. 2001). Las tecnologías empleadas en la actualidad, generalmente se enfocan en reducir costos de producción y no en los beneficios netos del cultivo, como por ejemplo el manejo de genotipos que pueden resultar en materiales que se adapten a las condiciones del lugar (Linares 2004). Por consiguiente, los procesos de investigación en este campo, se hacen vitales para buscar nuevos cultivares que permitan a los agricultores hacer frente a potenciales desafíos como plagas en las plantas, el cambio climático; así como el fortalecimiento de la dieta alimenticia de la población (FAO 2006).

El presente trabajo de investigación tuvo como objetivo evaluar la adaptabilidad de seis cultivares de chile dulce (Capsicum annum) a las condiciones ambientales generadas en condiciones de invernadero.

\section{MATERIALES Y MÉTODOS}

La actividad se realizó en la Estación Experimental Enrique Jiménez Núñez, en Cañas, provincia de Guanacaste (14 msnm), durante el periodo comprendido de octubre del 2014 a mayo del 2015. El sitio se encuentra dentro de la zona de vida del bosque tropical seco en la coordenada E00411952 y N00258587.
Los tratamientos fueron dispuestos en el diseño de bloques completos al azar, con cuatro repeticiones. La unidad experimental fue de 20 plantas por parcela de cada cultivar F1 (Cuadro 1). La dimensión de la parcela experimental fue de $4 \mathrm{~m}$ de largo por 0,40 $\mathrm{m}$ de ancho. Se utilizaron cuatro canaletas de $0,4 \mathrm{~m}$ de ancho 
por 0,2 $\mathrm{m}$ de altura con sustrato hidropónico (piedra volcánica). La distancia entre plantas fue de $0,2 \mathrm{~m}$ dentro de la canaleta y 1,4 m entre el pasillo, para una densidad de 35714 plantas por hectárea.

Cuadro 1. Cultivares utilizados en el estudio y su procedencia. EEEJN, 2015

\begin{tabular}{|l|l|l|}
\hline \multicolumn{1}{r|}{ Código } & \multicolumn{1}{|c|}{ Cultivar (F1) } & Procedencia \\
\hline CTT 01-14 & FBM 2 & UCR \\
\hline CTT 02-14 & FBM 9 & UCR \\
\hline CTT 03-14 & FBM 10 & UCR \\
\hline CTT 04-14 & FBM 11 & UCR \\
\hline CTT 05-14 & FBM 12 (Dulcitico) & UCR \\
\hline${ }^{*}$ CTT 06-14 & Nathalie & ROGERS \\
\hline
\end{tabular}

* Testigo

El estudio se efectuó en la segunda nave de un invernadero multicapilla orientado norte-sur. EI techo estuvo cubierto con plástico transparente de polietileno de 150 micras de espesor (Figura 1).

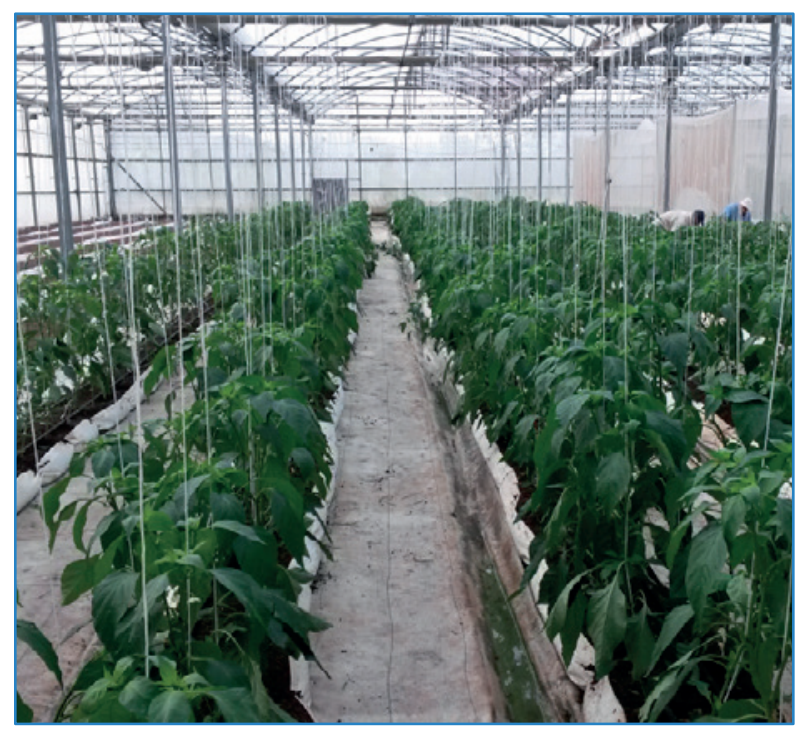

Figura 1. Ensayo de chile dulce en invernadero multicapilla. EEEJN, 2015.
La semilla se sembró en bandejas con sustrato inerte y las plántulas se trasplantaron al invernadero a los 25 días después de la siembra. El riego en las canaletas se realizó mediante un sistema presurizado con cintas de goteo, añadiéndose al sistema $180 \mathrm{~kg}$ de $\mathrm{N}, 60 \mathrm{~kg} \mathrm{P} \mathrm{O}_{5}, 180 \mathrm{~kg} \mathrm{~K} \mathrm{O}_{5}$, $160 \mathrm{CaO}$ y $50 \mathrm{~kg}$ de $\mathrm{MgO}$ por hectárea respectivamente. El manejo fitosanitario se realizó según la presión de enfermedades y plagas en el momento.

Se utilizó un muestreo probabilístico al azar, con un factor de muestreo para $Z_{a}{ }^{2}$ de $(1,96)^{2}$ y un error máximo permitido del $14 \%$. Al ser los híbridos de chile dulce $\mathrm{F} 1$, la proporción de la población que posee la característica deseada se estima que es cercano al $95 \%(p=0,95)$, resultando una muestra de 6 plantas por parcela útil.

Como variables de respuesta se evaluó en una muestra de seis plantas:

a. Rendimiento: se cuantificó el rendimiento por parcela de muestreo, según número y peso promedio de frutos.

b. Calidad (norma comercial): Se clasificó la producción según la longitud de fruta, siendo la primera calidad (frutos $\geq 10 \mathrm{~cm}$ ), segunda calidad (frutos $<10 \mathrm{~cm}$ y rechazo o no comerciales (con defectos o quemas de fruto)

c. Tipo de fruta y color a la maduración.

d. Número de cosechas.

e. Altura de la planta (m).

f. Producción por hectárea (t).

Los datos obtenidos fueron sometidos al análisis de varianza y las medias separadas mediante la prueba de Duncan a un nivel de significancia del $5 \%$. Además, se registraron las condiciones ambientales: temperatura, humedad relativa y radiación a las que estuvieron sometidas las plantas, realizando tres mediciones diarias (7 a.m.; 9 a.m. y 1 p.m.) una vez por semana, durante todo el ciclo productivo. Para determinar la rentabilidad de cada tratamiento, se realizó un análisis económico, obteniendo la utilidad bruta y la relación beneficio-costo. 


\section{RESULTADOS Y DISCUSIÓN}

\section{Características agronómicas}

El cultivar que obtuvo la mayor altura promedio por planta fue FBM 11 con 2,5 m, mostrando diferencias significativas con FBM 9, Dulcitico y Nathalie. Alturas por debajo de los $2,5 \mathrm{~m}$ facilitan las labores de cosecha, poda y deshijas, ya que no se tiene que recurrir al uso de andamios o escaleras, lo cual agiliza las prácticas de manejo.
A Dulcitico y FBM 10 se les realizó 13 cosechas, mientras que al resto de los cultivares solamente 12. Esta cantidad de cosechas coincide con la investigación realizada en este mismo cultivo por el INTA en el año 2013 (Ramírez et al. 2013). Todos los materiales presentaron frutos de tipo cónico con tres lóbulos y con maduración a rojo, las cuales son las más apetecidas por el mercado nacional (Cuadro 2).

Cuadro 2. Altura de la planta $(m)$, número de cosechas y características de los frutos de chile dulce. EEEJN, 2015.

\begin{tabular}{|c|c|c|c|c|c|}
\hline Cultivar & $\begin{array}{l}\text { Altura de } \\
\text { planta (m) }\end{array}$ & $\mathbf{N}^{\circ}$ de cosechas & $\begin{array}{l}\text { Tipo de } \\
\text { fruto }\end{array}$ & $\begin{array}{l}N^{\circ} \text { de } \\
\text { lóbulos }\end{array}$ & $\begin{array}{l}\text { Color al } \\
\text { madurar }\end{array}$ \\
\hline FBM 2 & $2,3 \mathrm{bc}$ & 12 & Cónico & 3 & Rojo \\
\hline FBM 9 & $1,9 \mathrm{~b}$ & 12 & Cónico & 3 & Rojo \\
\hline FBM 10 & $2,1 \mathrm{bc}$ & 13 & Cónico & 3 & Rojo \\
\hline FBM 11 & $2,5 \mathrm{c}$ & 12 & Cónico & 3 & Rojo \\
\hline Dulcitico & $2 b$ & 13 & Cónico & 3 & Rojo \\
\hline Nathalie & $1,4 \mathrm{a}$ & 12 & Cónico & 3 & Rojo \\
\hline
\end{tabular}

Letras distintas indican diferencias significativas $(p \leq 0,05)$

En Costa Rica la clasificación de la calidad del chile dulce no solo se define por el aspecto externo de la fruta; sino también, por la longitud de la misma. El cultivar FBM-10 fue el que obtuvo el mayor número de frutos en primera calidad en la muestra, con 94 unidades para un promedio de 15,6 unidades por planta, mostrando diferencias significativas solamente con FBM 11. El mayor peso promedio por frutos de primera lo alcanzó FBM 11 con 67,8 g, presentando diferencias significativas con todos los cultivares a excepción de FBM 2 y Nathalie. Con relación a la longitud de los frutos, todos los cultivares estuvieron cercanos al límite inferior de la categoría de primera calidad que es de $10 \mathrm{~cm}$ (Cuadro 3). El mayor rendimiento por parcela experimental en esta misma categoría lo obtuvo FBM 10 con $5951,7 \mathrm{~g}$, para un promedio de $991,9 \mathrm{~g}$ por planta, presentando diferencias significativas únicamente con FBM 11.

El cultivar FBM 10 alcanzó el mayor número de frutos de segunda calidad en el área de muestreo con 121 unidades, para un promedio de 20,2 unidades por planta, mostrando diferencias significativas excepto con Dulcitico. El híbrido Nathalie fue el que obtuvo el mayor peso promedio dentro de esta categoría con $48,8 \mathrm{~g}$, presentando diferencias significativas únicamente con FBM 9 (Cuadro 3). El rango de longitud promedio de frutos de segunda categoría se situó entre los $8 \mathrm{~cm}$ (FBM 10) hasta los $8,4 \mathrm{~cm}$ (FBM 9). El cultivar FBM 10 que fue el de mayor producción de frutos de segunda alcanzó el mayor rendimiento en el área de muestreo con $5418 \mathrm{~g}$, para un promedio de $903 \mathrm{~g}$ por planta, difiriendo significativamente con FBM 2, FBM 9 y FBM 11.

En lo que se refiere a los frutos de tercera categoría, FBM-9 presentó la mayor cantidad con cinco unidades, existiendo diferencias significativas con todos los cultivares a excepción de Dulcitico, con 4,7 unidades (Cuadro 3). Los pesos promedios de los frutos estuvieron entre los 8,75 a los $38,2 \mathrm{~g}$ entre todos los materiales. El cultivar FBM 9 obtuvo en el área de muestreo la mayor cantidad en peso de frutos de tercera con $194,7 \mathrm{~g}$, mostrando diferencias significativas con el resto de los materiales, salvo con Dulcitico $(171,7 \mathrm{~g})$. 
El cultivar FBM 10 obtuvo el mayor rendimiento por hectárea con 68,1 toneladas, no difiere del Dulcitico, el cual obtuvo 56,87 t/ha, pero si del resto de los materiales. Los rendimientos obtenidos con FBM 10 están 27,4 toneladas por hectárea por debajo del reportado por Echandi 2012, con híbrido Dulcitico en la provincia de Alajuela, pero superan en 28,1 toneladas por hectárea a los alcanzados por el INTA en el año 2013, en la provincia de Guanacaste, con el híbrido Andes Cobayashi (Ramírez et al. 2013) y también supera en $1,3 \mathrm{~kg}$ por planta, el promedio nacional que está en $0,6 \mathrm{~kg}$ (Morales y Rodríguez 2010).

Cuadro 3. Clasificación de las características agronómicas de los cultivares según calidad y número de plantas muestreadas. EEEJN, 2015.

\begin{tabular}{|c|c|c|c|c|c|c|c|}
\hline Cal*. & Característica & FBM 2 & FBM 9 & FBM 10 & FBM 11 & Dulcitico & Nathalie \\
\hline \multirow{4}{*}{$1^{\mathrm{a}}$} & $\mathrm{N}^{\circ}$ de frutos/muestra & $74,0 \mathrm{ab}$ & $77,7 \mathrm{ab}$ & $94,0 \mathrm{~b}$ & 57,5 a & $74,7 \mathrm{ab}$ & $71,0 a b$ \\
\hline & Peso frutos (g) & $66,7 \mathrm{bc}$ & $60,5 \mathrm{a}$ & $63,1 a b$ & $67,8 c$ & $62,2 \mathrm{a}$ & 69,6 c \\
\hline & Longitud frutos (cm) & 10,9 & 10,9 & 10,7 & 10,9 & 10,7 & 10,9 \\
\hline & Rendimiento/muestra (g) & $4948,6 a b$ & $4722,6 \mathrm{ab}$ & 5951,7 b & 3931,1 a & $4662,5 \mathrm{ab}$ & $4917 \mathrm{ab}$ \\
\hline \multirow{4}{*}{$2^{\mathrm{a}}$} & $\mathrm{N}^{\circ}$ de frutos/muestra & 69,7 a & $82,5 a b$ & $121,0 \mathrm{c}$ & $69,7 \mathrm{a}$ & 110,7 bc & $83,0 a b$ \\
\hline & Peso frutos (g) & $46,0 \mathrm{ab}$ & $41,1 \mathrm{a}$ & $44,4 \mathrm{ab}$ & $45,0 a b$ & $42,4 a b$ & $48,8 \mathrm{~b}$ \\
\hline & Longitud frutos (cm) & 8,2 & 8,4 & 8,0 & 8,2 & 8,1 & 8,2 \\
\hline & Rendimiento/muestra (g) & 3228,6 a & 3352,4 a & $5418 b$ & 3053,9 a & $4719,2 a b$ & $4135 a b$ \\
\hline \multirow{3}{*}{$3^{a}$} & $\mathrm{~N}^{\circ}$ de frutos/muestra & $0,5 \mathrm{a}$ & $5,0 \mathrm{~b}$ & $2,0 \mathrm{a}$ & $1,3 \mathrm{a}$ & $4,7 \mathrm{~b}$ & $0,3 \mathrm{a}$ \\
\hline & Peso frutos (g) & 8,7 & 38,2 & 27,2 & 16,1 & 39,1 & 14,7 \\
\hline & Rendimiento/muestra (g) & $17,5 \mathrm{a}$ & $194,7 \mathrm{~b}$ & $71,7 \mathrm{a}$ & $48,7 \mathrm{a}$ & $171,7 \mathrm{~b}$ & $14,7 \mathrm{a}$ \\
\hline \multicolumn{2}{|c|}{ Rendimiento/ha, t } & $48,8 \mathrm{ab}$ & $49,2 \mathrm{a}$ & $68,1 \mathrm{c}$ & $41,9 \mathrm{a}$ & $56,9 \mathrm{bc}$ & $54,0 a b$ \\
\hline
\end{tabular}

Letras distintas indican diferencias significativas $(p \leq 0,05)$

${ }^{*}$ Cal. $=$ calidad

La Figura 2 muestra como el cultivar FBM 10 obtuvo el mayor rendimiento productivo por hectárea, tanto en los frutos de primera como de segunda categoría, con 35,43 y 32,25 toneladas por hectárea, respectivamente. El cultivar FBM
11 presentó el menor rendimiento de frutos de primera y segunda, con 23,40 y 18,18 toneladas por hectárea respectivamente, por último, FBM 9 obtuvo la mayor cantidad de frutos de tercera calidad con 1,2 toneladas por hectárea.

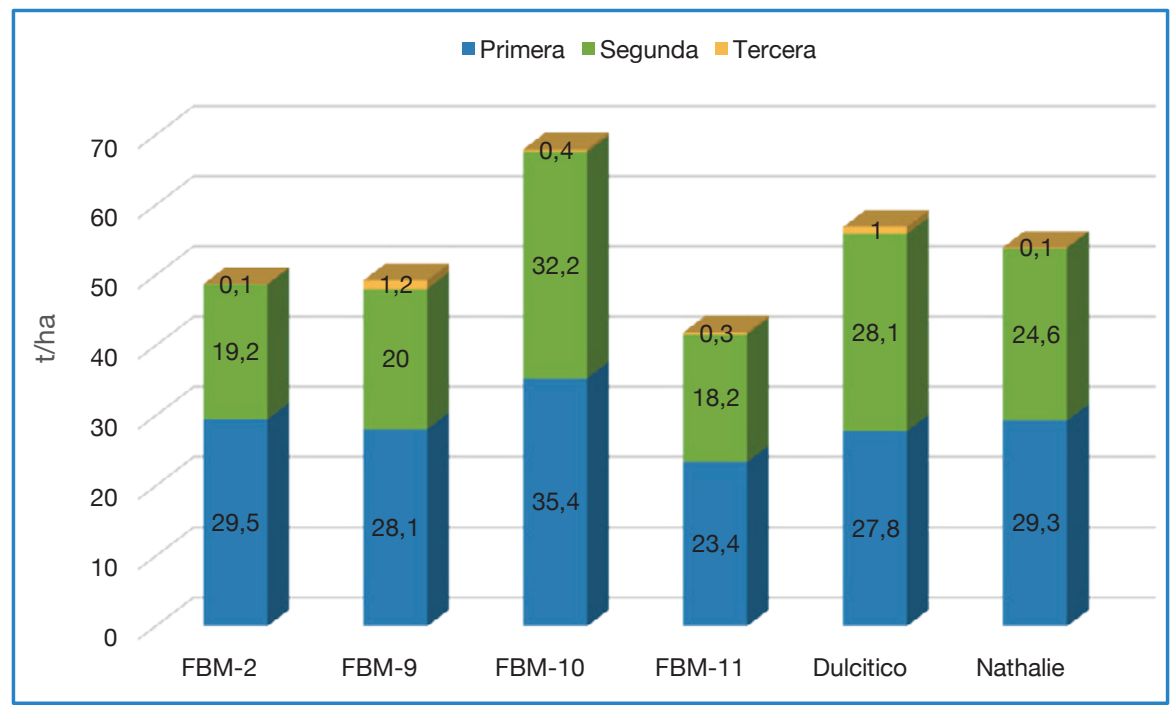

Figura 2. Rendimiento productivo (t/ha), según categoría comercial (primera, segunda y tercera calidad). EEEJN, 2015. 
Todos los materiales a excepción de Dulcitico, obtuvieron una mayor proporción de frutos de primera que de segunda calidad. El cultivar FBM 2 presentó el mayor porcentaje de frutos de primera con $60 \%$ y el menor porcentaje de frutos de segunda con un $39 \%$ y $1 \%$ de rechazo. El cultivar que obtuvo el mayor rendimiento productivo (FBM 10) $(68,1$ t/ha) presentó un $52 \%$ de frutos de primera, un $47,3 \%$ de frutos de segunda y un $0,6 \%$ de tercera (Figura 3 ).

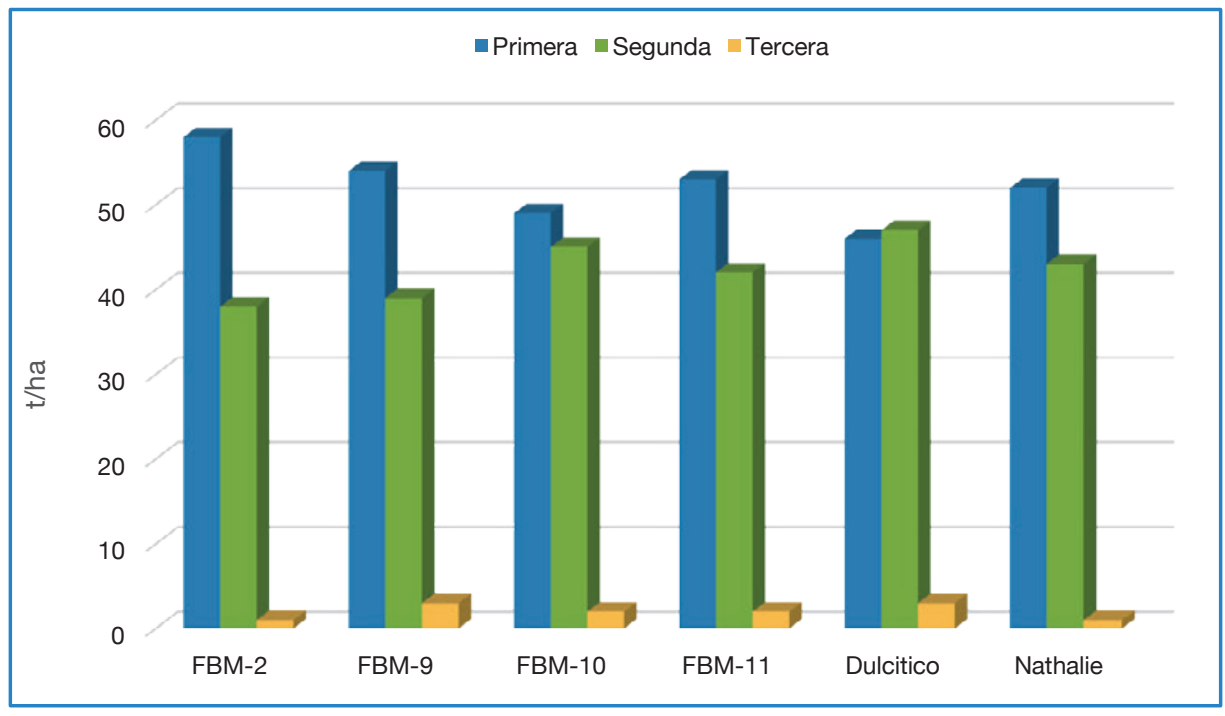

Figura 3. Clasificación de la producción (\%) según categoría (Primera, segunda y tercera) comercial para los cultivares de chile dulce. EEEJN, 2015.

\section{Monitoreo de los factores climáticos}

\section{Temperatura}

La menor temperatura promedio dentro del invernadero se presentó a las 7 a.m., con un valor de $27,1^{\circ} \mathrm{C}$ y una deviación estándar de 1,7. Posteriormente, a las 9 a.m. se presentó un incremento en la temperatura dentro del módulo llegando en promedio a los $32,3{ }^{\circ} \mathrm{C}$, con un salto térmico (Diferencia de temperatura entre el exterior y el interior del invernadero) de $2,1^{\circ} \mathrm{C}$ y una desviación estándar de 1,4 (Cuadro 4). Estas mediciones nos indican que al menos hasta las 9 a.m., la temperatura, al no superar los $35^{\circ} \mathrm{C}$ (límite superior para una normal actividad fotosintética) no estaría afectando las principales funciones fisiológicas de las plantas, las cuales son: absorción de agua y elementos minerales por las raíces, la transpiración del vapor de agua por las hojas, junto con la fotosíntesis y la respiración (Berninger 1989).
Por otra parte, la máxima temperatura dentro del invernadero se presentó a la 1 pm, promediando $35,1^{\circ} \mathrm{C}$, con un salto térmico de $2{ }^{\circ} \mathrm{C}$ y una desviación estándar de 1,4, lo que nos indica que hubo meses como octubre, noviembre, febrero, marzo, abril y mayo que presentaron temperaturas mayores al límite superior del rango de eficiencia fotosintética para el cultivo de chile dulce que es de $35^{\circ} \mathrm{C}$, afectando el funcionamiento de la planta con cierres parciales o totales de los estomas, lo que disminuye la traspiración y la conversión de foto asimilados. Esta situación limita la capacidad de producir frutos y ocasiona una senescencia más temprana de la planta, lo que disminuye el número de cosechas, que para este ensayo tuvo un máximo de 13. 
Cuadro 4. Registros de la temperatura $\left({ }^{\circ} \mathrm{C}\right.$ ) en el interior y exterior del invernadero. (Octubre 2014 a mayo 2015). EEEJN, 2015.

\begin{tabular}{|c|c|c|c|c|c|c|}
\hline \multirow[b]{2}{*}{ Mes } & \multicolumn{2}{|c|}{7 a.m. } & \multicolumn{2}{|c|}{9 a.m. } & \multicolumn{2}{|c|}{1 p.m. } \\
\hline & $\begin{array}{l}\text { Temperatura } \\
\text { externa, }{ }^{\circ} \mathbf{C}\end{array}$ & $\begin{array}{l}\text { Temperatura } \\
\text { interna, }{ }^{\circ} \mathbf{C}\end{array}$ & $\begin{array}{c}\text { Temperatura } \\
\text { externa, }{ }^{\circ} \mathbf{C}\end{array}$ & $\begin{array}{l}\text { Temperatura } \\
\text { interna, }{ }^{\circ} \mathbf{C}\end{array}$ & $\begin{array}{l}\text { Temperatura } \\
\text { externa, }{ }^{\circ} \mathbf{C}\end{array}$ & $\begin{array}{c}\text { Temperatura } \\
\text { interna, }{ }^{\circ} \mathrm{C}\end{array}$ \\
\hline Octubre & 26,3 & 28,1 & 30,4 & 33,6 & 33,7 & 36,0 \\
\hline Noviembre & 24,9 & 26,8 & 29,1 & 32,7 & 32,5 & 35,3 \\
\hline Diciembre & 25,4 & 26,1 & 28,9 & 30,8 & 30,6 & 32,9 \\
\hline Enero & 26,2 & 26,3 & 28,6 & 30,4 & 30,5 & 33,1 \\
\hline Febrero & 24,5 & 24,3 & 29,6 & 31,3 & 34,4 & 36,4 \\
\hline Marzo & 28,1 & 29,0 & 31,4 & 34,1 & 33,1 & 35,3 \\
\hline Abril & 28,9 & 29,4 & 31,3 & 32,5 & 33,9 & 35,2 \\
\hline Mayo & 26,3 & 26,6 & 32,4 & 33,2 & 36,2 & 36,7 \\
\hline Promedio & 26,3 & 27,1 & 30,2 & 32,3 & 33,1 & 35,1 \\
\hline Desv. Est. & 1,5 & 1,7 & 1,4 & 1,4 & 1,9 & 1,4 \\
\hline
\end{tabular}

\section{Humedad relativa}

La mayor higrometría dentro del invernadero se presentó a las 7 a.m. con un promedio de $75,7 \%$ y una desviación estándar de 7,9 (Cuadro 5). Estos contenidos de vapor de agua cercanos a la saturación, se deben a la disminución de la temperatura en la noche, que provoca una condensación del vapor de agua. A las 9 a.m. se presentó una disminución de la humedad dentro del recinto en 11,7 unidades, por efecto del incremento de la temperatura, que en promedio para esta hora fue de $32,3{ }^{\circ} \mathrm{C}$. Aunque la humedad ambiental no influye directamente en la fotosíntesis, su papel es indirecto a través de la apertura estomática, por lo que los niveles antes descritos en ese lapso de la mañana no debieran limitar la transpiración de la planta.
A la 1 p.m. se obtuvo en promedio la menor humedad relativa dentro del módulo con un $54,2 \%$, sin que existiera una gran fluctuación de los valores con respecto al promedio, ya que la desviación estándar fue de 5,1. Aunque la higrometría del aire en este punto del día presentó sus niveles más bajos, cabe destacar que superó la humedad promedio externa, la cual fue de $51,3 \%$, lo que evidencia que las plantas tuvieron algún nivel de transpiración, aportando vapor de agua al ambiente, lo que significa que las raíces absorbieron agua y minerales en las horas más calientes del día. Aun así, niveles por debajo del $70 \%$ de humedad son perjudiciales para el cuajado del fruto y provoca el aborto floral, lo que puede explicar la razón por la cual, el rendimiento obtenido en esta prueba sea 38,6 toneladas menor al obtenido por Echandi 2012.

Cuadro 5. Registros de humedad relativa (\%) en el interior y exterior del invernadero. (Octubre 2014 a mayo 2015). EEEJN, 2015.

\begin{tabular}{|c|c|c|c|c|c|c|}
\hline \multirow[b]{2}{*}{ Mes } & \multicolumn{2}{|c|}{7 a.m. } & \multicolumn{2}{|c|}{9 a.m. } & \multicolumn{2}{|c|}{1 p.m. } \\
\hline & $\begin{array}{l}\text { Humedad } \\
\text { externa, \% }\end{array}$ & $\begin{array}{l}\text { Humedad } \\
\text { interna, \% }\end{array}$ & $\begin{array}{l}\text { Humedad } \\
\text { externa, \% }\end{array}$ & $\begin{array}{l}\text { Humedad } \\
\text { interna, \% }\end{array}$ & $\begin{array}{c}\text { Humedad } \\
\text { externa, \% }\end{array}$ & $\begin{array}{l}\text { Humedad } \\
\text { interna, \% }\end{array}$ \\
\hline Octubre & 84,3 & 79,7 & 71,1 & 66,7 & 62,2 & 57,5 \\
\hline Noviembre & 88,4 & 88,2 & 73,9 & 71,8 & 58,6 & 57,1 \\
\hline Diciembre & 67,4 & 73,4 & 59,2 & 66,6 & 55,0 & 58,3 \\
\hline Enero & 65,9 & 72,3 & 59,4 & 66,3 & 55,5 & 60,8 \\
\hline Febrero & 72,1 & 77,1 & 58,4 & 63,4 & 40,5 & 47,1 \\
\hline Marzo & 61,6 & 67,1 & 51,7 & 54,5 & 46,2 & 52,6 \\
\hline Abril & 59,7 & 64,6 & 52,0 & 57,8 & 47,6 & 52,9 \\
\hline Mayo & 81,8 & 83,0 & 55,3 & 64,9 & 45,1 & 47,0 \\
\hline Promedio & 72,6 & 75,7 & 60,1 & 64 & 51,3 & 54,2 \\
\hline Des. Est. & 10,9 & 7,9 & 8,2 & 5,5 & 7,5 & 5,1 \\
\hline
\end{tabular}


El comportamiento de la temperatura y la humedad relativa, aunque no es inversamente proporcional, se encontró un estrecho ligamen durante los meses en que se llevó a cabo el experimento. Conforme la temperatura promedio diaria aumentó dentro del invernadero, a partir del mes de marzo $\left(32,8^{\circ} \mathrm{C}\right)$ la humedad relativa se situó en los niveles más bajos, cercanos al 57 \% (Figura 4). Estos niveles de humedad posibilitan el uso de sistemas de humidificación para aumentar la higrometría del aire, con el fin de disminuir la temperatura y generar un mejor ambiente para que las plantas realicen eficientemente su metabolismo.

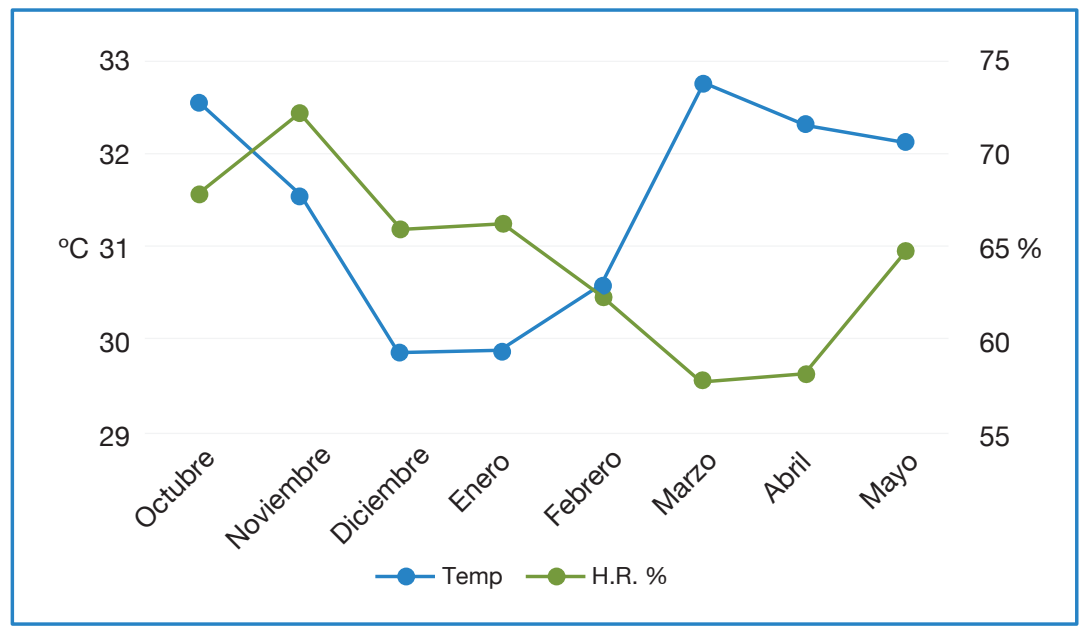

Figura 4. Promedio diario de las variables climáticas temperatuta y humedad relativa dentro del invernadero, durante los meses del experimento. (Octubre 2014 a mayo 2015). EEEJN, 2015.

\section{Radiación}

Los niveles promedio de la Radiación Fotosintéticamente Activa (PAR) dentro del invernadero, una vez traspasada la cobertura plástica a las 7 a.m., 9 a.m. y 1 p.m. fueron de 26, 88 y 98 watts $/ \mathrm{m}^{2}$, respectivamente. Esto representa una reducción de un $52 \%$ con respecto a la radiación registrada fuera del recinto (Cuadro 6). La disminución del paso de la luz se debió a la presencia de una pantalla de sombreo tipo luminet de $50 \%$ sombra, en los meses de mayo y abril para evitar quemadura de frutos por el sol. En la mayoría de cultivos C3 como el chile dulce, la saturación del sistema fotosintético se alcanza alrededor de los 90 watts $/ \mathrm{m}^{2}$, lo que sugiere que la cantidad de radiación que llegó a las plantas en estudio fue óptima para desarrollar el proceso fotosintético desde tempranas horas de la mañana. (Langhams et al. 1997).

La deviación estándar para esta variable evidencia que existe una variación importante durante las tres horas (7 a.m.; 9 a.m. y 1 p.m.) que se realizó las lecturas con valores de 15, 31 y 23 respectivamente. Estas fluctuaciones son un reflejo de la dinámica ambiental presente en la época seca en la Región Chorotega, lo que demuestra que el manejo de la luz por medio de coberturas plásticas y sombras es imprescindible para buscar mejorar el rendimiento productivo y la calidad de la producción. 
Cuadro 6. Registros de radiación fotosintéticamente activa (PAR) (watts $\left./ \mathrm{m}^{2}\right)$ en el interior y exterior del invernadero (EEEJN, octubre 2014 a mayo 2015).

\begin{tabular}{|c|c|c|c|c|c|c|}
\hline \multirow[b]{2}{*}{ Mes } & \multicolumn{2}{|c|}{7 a.m. } & \multicolumn{2}{|c|}{9 a.m. } & \multicolumn{2}{|c|}{1 p.m. } \\
\hline & $\begin{array}{l}\text { Radiación } \\
\text { externa, PAR }\end{array}$ & $\begin{array}{c}\text { Radiación } \\
\text { interna, PAR }\end{array}$ & $\begin{array}{c}\text { Radiación } \\
\text { externa, PAR }\end{array}$ & $\begin{array}{c}\text { Radiación } \\
\text { interna, PAR }\end{array}$ & $\begin{array}{c}\text { Radiación } \\
\text { externa, PAR }\end{array}$ & $\begin{array}{c}\text { Radiación } \\
\text { interna, PAR }\end{array}$ \\
\hline Octubre & 91 & 51 & 166 & 100 & 196 & 135 \\
\hline Noviembre & 63 & 36 & 211 & 136 & 159 & 83 \\
\hline Diciembre & 65 & 30 & 177 & 98 & 144 & 79 \\
\hline Enero & 13 & 8 & 156 & 76 & 172 & 96 \\
\hline Febrero & 38 & 12 & 173 & 91 & 227 & 120 \\
\hline Marzo & 82 & 35 & 193 & 108 & 211 & 116 \\
\hline Abril & 62 & 20 & 154 & 63 & 242 & 92 \\
\hline Mayo & 71 & 16 & 137 & 34 & 229 & 66 \\
\hline Promedio & 60 & 26 & 171 & 88 & 198 & 98 \\
\hline Desv. Est. & 25 & 15 & 23 & 31 & 36 & 23 \\
\hline
\end{tabular}

\section{Análisis económico}

\section{Costos}

El rubro que tiene el mayor costo económico es el invernadero, con 1,5 millones de colones por $1000 \mathrm{~m}^{2}$ de área, suponiendo una depreciación de la estructura a 10 años, lo que significa un $50 \%$ de la inversión inicial. El segundo mayor egreso lo representa la mano de obra con 0,67 millones de colones por cada $1000 \mathrm{~m}^{2}$ (Cuadro 7). Este tipo de actividad necesita un personal especializado, por lo que se pueden generar empleos permanentes que impacten socialmente a zonas con pocas posibilidades de empleo como Guanacaste. Además, este desglose de los costos nos indica que, para mejorar la competitividad de la actividad de chile dulce, se deben valorar otras opciones de ambiente protegido, con menor costo que los invernaderos.

Cuadro 7. Costos de insumos utilizados en $1000 \mathrm{~m}^{2}$ de chile dulce en invernadero. EEEJN, 2015

\begin{tabular}{|c|c|c|c|c|}
\hline Rubro & $\begin{array}{l}\text { Unidad de } \\
\text { medida }\end{array}$ & $\begin{array}{l}\text { cantidad } \\
\left(1000 \mathrm{~m}^{2}\right)\end{array}$ & $\begin{array}{c}\text { valor/unidad } \\
\text { (millones } \$ \text { ) }\end{array}$ & $\begin{array}{c}\text { Total } \\
\text { (millones } \phi)\end{array}$ \\
\hline Mano de obra & Jornales & 72 & 0,0093 & 0,67 \\
\hline Cuido almácigos & $\mathrm{N}^{\circ}$ bandejas & 36,5 & 0,0004 & 0,01 \\
\hline Cintas de goteo & Rollo/2200m & 1 & 0,1 & 0,10 \\
\hline Solución nutritiva & $\mathrm{m}^{3}$ & 126 & 0,004 & 0,50 \\
\hline Semilla & Unitario & 3571,4 & 0,000025 & 0,09 \\
\hline Funguicidas & Kilogramos & 1 & 0,002 & 0,002 \\
\hline Insecticidas & Litros & 1 & 0,001 & 0,001 \\
\hline Invernadero y equipo de riego* & $\mathrm{m}^{2}$ & 1000 & 0,0015 & 1,50 \\
\hline Sustrato** & $\mathrm{m}^{3}$ & 58,3 & 0,0013 & 0,08 \\
\hline Contenedor ${ }^{\star \star *}$ & $\mathrm{~m}$ & 1071,4 & 0,00011 & 0,12 \\
\hline Total & & & & 3,07 \\
\hline
\end{tabular}

*Depreciación a 10 años.

** Depreciación a 6 años

*** Depreciación a 6 años 


\section{Flujo de caja}

El cultivar de chile dulce que obtuvo la mayor utilidad neta fue el FBM 10 con 2,11 millones de colones por cada $1000 \mathrm{~m}^{2}$. Esto se debió a que obtuvo los mayores ingresos totales de frutas de primera y segunda calidad, con 3,08 y 2,10 millones de colones respectivamente, superando en ingresos al cultivar testigo (Nathalie) por 1,08 millones de colones (Cuadro 8). Todos los cultivares obtuvieron una relación beneficio costo superior a 1, lo que indica que todos presentan ganancias; aunque se debe destacar que el mayor valor lo obtuvo FBM 10 con 1,69, lo que significa que, por cada colón invertido, se recupera ese colón y genera 0,69 colones de ganancia, demostrándose que esta actividad es rentable para productores o inversionistas.

Cuadro 8. Flujo de caja para $1000 \mathrm{~m}^{2}$ de chile dulce en invernadero. EEEJN, 2015.

\begin{tabular}{|c|c|c|c|c|c|c|c|c|}
\hline \multirow{3}{*}{ Cultivar } & \multicolumn{4}{|c|}{ Ingresos } & \multirow{3}{*}{$\begin{array}{l}\text { Ingreso total } \\
\text { (millones de } \\
\text { colones) }\end{array}$} & \multirow{3}{*}{$\begin{array}{c}\text { Egreso total } \\
\text { Costo de } \\
\text { insumos } \\
\text { (millones } \phi \text { ) }\end{array}$} & \multirow{3}{*}{$\begin{array}{c}\text { Saldo } \\
\text { (millones } \phi \text { ) }\end{array}$} & \multirow{3}{*}{$\begin{array}{c}\text { Relac. } \\
\text { B/C }\end{array}$} \\
\hline & \multicolumn{2}{|c|}{ Primera calidad } & \multicolumn{2}{|c|}{ Segunda calidad } & & & & \\
\hline & $\begin{array}{c}\text { Rendimiento } \\
\left(\mathrm{t} / 1000 \mathrm{~m}^{2}\right)\end{array}$ & $\begin{array}{c}\text { Ingreso } \\
\text { (millones de } \\
\text { colones) }\end{array}$ & $\begin{array}{c}\text { Rendimiento } \\
\left(\mathrm{t} / 1000 \mathrm{~m}^{2}\right)\end{array}$ & $\begin{array}{c}\text { Ingreso } \\
\text { (millones de } \\
\text { colones) }\end{array}$ & & & & \\
\hline FBM 2 & 2,95 & 2,56 & 1,92 & 1,25 & 3,81 & 3,07 & 0,74 & 1,24 \\
\hline FBM 9 & 2,81 & 2,44 & 2,00 & 1,30 & 3,75 & 3,07 & 0,68 & 1,22 \\
\hline FBM 10 & 3,54 & 3,08 & 3,22 & 2,10 & 5,18 & 3,07 & 2,11 & 1,69 \\
\hline FBM 11 & 2,34 & 2,03 & 1,82 & 1,19 & 3,22 & 3,07 & 0,15 & 1,05 \\
\hline Dulcitico & 2,78 & 2,41 & 2,81 & 1,83 & 4,25 & 3,07 & 1,18 & 1,38 \\
\hline Nathalie & 2,93 & 2,55 & 2,46 & 1,61 & 4,15 & 3,07 & 1,08 & 1,35 \\
\hline
\end{tabular}

Chile dulce de primera calidad: $\$ 869,665 /$ t.

Chile dulce de segunda calidad: $\$ 652,174 / \mathrm{t}$.

(Fuente: PIMA CENADA 2015).

\section{LITERATURA CITADA}

Berninger, E. 1989. Cultures florales de serre en zone mediterranéenne francaise: Eléments climatiques et physiologiques. Paris. INRA, PHM-Revue Horticole. 23 p.

Echandi, C. 2012. Híbridos promisorios de chile dulce para la zona media-alta de Costa Rica. Boletín Técnico Quincenal de la Estación Experimental Agrícola Fabio Baudrit, Universidad de Costa Rica. Alajuela, Costa Rica. p. 4-7.

FAO (Organización de las Naciones Unidas para la Agricultura y la Alimentación). 2006. Los recursos genéticos en la agricultura: clave para la seguridad alimentaria. (en línea) Roma, Italia. 2 p. Consultado 16 set. 2014. Disponible en: http://www.fao.org/ NEWSROOM/es/news/2006/1000316/index.html
FAO (Organización de las Naciones Unidas para la Agricultura y la Alimentación). 2014. Production de produits alimentaires et agricoles (en línea). Roma, Italia. 4 p. Consultado 05 ago. 2014. Disponible en: http://faostat.fao.org/site/339/default.aspx

Giménez, F; Lúquez, J; Suárez, J. 2001. Estabilidad y adaptabilidad de cultivares de soja para rendimiento en el sudeste de la provincia de Buenos Aires. (en línea). Rev. Fac. Agron., La Plata, Argentina 104(2) 3-103. Consultado 14 set. 2014. Disponible en: http://www2.agro.unlp.edu. ar/uploads/R/104_93_103.pdf 
Linares, L. 2004. Comportamiento de variedades de chile dulce (Capsicum annum) en la región occidental de El Salvador. Agronomía Mesoamericana. 15(1):2-3.

Ministerio de Salud, Costa Rica; MEIC (Ministerio de Economía, Industria y Comercio, Costa Rica); MAG (Ministerio de Agricultura y Ganadería). 2007. Reglamento técnico para el consumo fresco de chile dulce (en línea). Diario Oficial La Gaceta $N^{\circ}$ 101, San José, Costa Rica; 28 may. 2007. p. 2-8. Consultado 4 abr. 2015. Disponible en: www. imprentanacional.go.cr/gaceta/

Morales, D; Rodríguez, R. 2010. Estudio de viabilidad técnica y económica para el desarrollo de opciones de cosecha de lluvia y manejo adecuado en sistemas de riego en la producción agropecuaria. Alternativas rentables productivas por región. Guanacaste, Costa Rica. Ministerio de Agricultura y Ganadería (MAG). p.14-18. Consultoría SP-16-2009.

Orellana, F; Escobar, J; Morales, A; Méndez, I; Cruz, R; Castellón, M. 2012.Guía Técnico Cultivo de Chile Dulce. San Salvador, El Salvador. Centro Nacional de tecnología agropecuaria y Forestal (CENTA). (en línea). 4 p. Consultado 08 set. 2014. Disponible en http://www.centa.gob.sv/docs/guias/hortalizas/ Guia\%20Chile.pdf

PIMA (Programa Integral de Mercadeo Agropecuario, Costa Rica); CENADA (Centro Nacional de Abastecimiento y Distribución de Alimentos, Costa Rica). 2015. (en línea). Boletín setiembre 2015 de volúmenes y precios de mayorista a minoristas San José, Costa Rica. 1 p. Consultado 01 set. 2015. Disponible en http:// pima.go.cr/AP/CM/PG5028L1/PIMA-PROGRAMAINTEGRAL-DE-MERCADO-AGRO PECUARIO.aspx

PIMA (Programa Integral de Mercadeo Agropecuario, Costa Rica); CENADA (Centro Nacional de Abastecimiento y Distribución de Alimentos, Costa Rica). 2013. Tendencias de Consumo de Frutas, Hortalizas, Pesado y Mariscos en las familias de Costa Rica. (en línea) Heredia, Costa Rica. p. 38-42. Consultado el: 13 set. 2014. Disponible en: http://pima.go.cr/AP/CM/ PG1019L1/PIMA-PROGRAMA-INTEGRAL-DEMERCADO-AGROPECUARIO.aspx
Ramírez, R; Aguilar, J; Meza, L. 2013. Evaluación del rendimiento y adaptabilidad de nueve cultivares de chile dulce (Capsicum annum) bajo cobertura plástica en la Región Chorotega. San José, Costa Rica, INTA. Informe de archivos Técnicos del INTA. p 11-13.

Samaniego, R. 2006. Efecto de la producción orgánica y convencional de chile dulce (Capsicum annum) bajo invernadero sobre el componente planta-suelo en el cantón de Alfaro Ruiz. (en línea) Tesis MSc. Alajuela, Costa Rica. CATIE. 92 p. Consultado 12 set. 2014. Disponible en: http:// orton.catie.ac.cr/REPDOC/A0983E/A0983E.PDF

Urban, L. 1997. Introduction a la production sous serre. In: Castilla, N. Invernaderos de plástico. Madrid. España, Editorial Mundi-Prensa. p. 147-158. 